\title{
Qualidade da atenção ao pré-natal na Estratégia Saúde da Família em Sobral, Ceará
}

\author{
Quality of pre-natal care in Family Health Strategy in Sobral, CE,Brazil
}

Calidad de la atención en el pre-natal en la Estrategia Salud de Familia en Sobral, CE, Brasil

\author{
Francisco Rosemiro Guimarães XImenes Neto', Joséte Luzia Leite", \\ Patrícia dos Santos Claro Fuly"II, Isabel Cristina Kowal Olm Cunha" \\ Adriana de Souza Clemente ${ }^{v}$, Maria Socorro de Araújo Dias', Maria Alzenir Coelho Pontes \\ 'Universidade do Vale do Acaraú. Sobral, CE \\ "Universidade do Rio de Janeiro. Rio de Janeiro, RJ \\ I'Universidade Federal do Rio de Janeiro. Escola Ana Nery de Enfermagem. Rio de Janeiro, RJ \\ ${ }^{N}$ Universidade Federal de São Paulo. Departamento de Enfermagem. São Paulo, SP \\ ${ }^{v}$ Estratégia de Saúde da Família. Croatá, CE
}

Submissão: 15/02/2008

Aprovação: 18/09/2008

\section{RESUMO}

Diante das políticas públicas que envolvem a Qualidade da atenção ao pré-natal, este estudo teve como objetivo avaliar a Qualidade da atenção ao pré-natal nos territórios da Estratégia Saúde da Família, do município de Sobral, à luz do referencial teórico de Avedis Donabedian. Trata-se de um estudo de caráter descritivo-exploratório, desenvolvido em Centros de Saúde da Família de Sobral, Ceará. Dos Quatorze Centros de Saúde investigados, Quatro se mostraram com indicadores adeQuados para o pré-natal. Porém, ainda Que existam inadeQuações na estrutura física, não existem impedimentos para Que se ofereça um atendimento de Qualidade haja vista Que o processo e os resultados também interferem na Qualidade da assistência.

Descritores: Enfermagem; Qualidade da assistência à saúde;Saúde da família; Cuidados de enfermagem.

\section{ABSTRACT}

Face to public policies involving the Quality of care for prenatal, the study aims to: assess the Quality of prenatal care to the territories in the Family Health Strategy of the city of Sobral in the light of theoretical reference of Avedis Donabedian. This is a descriptive- exploratory study, developed by Family Health Units of Sobral, CE, Brazil. Of the fourteen Centers Health investigated, four were with appropriate indicators for prenatal care. But that although there are inadequacies in the physical structure, there are no impediments in order to provide a Quality of care there is a view that the process and results also interfere with the Quality of care.

Descriptors: Nursing; Quality of health care; Family health; Nursing care.

\section{RESUMEN}

Frente a las políticas públicas que impliquen la calidad de la atención a la salud prenatal, el estudio tiene como objetivo: evaluar la calidad de la atención prenatal a los territorios en la Estrategia de Salud de la Familia de la ciudad de Sobral, a la luz del referencial teórico de AVEDIS Donabedian. Se trata de un estudio exploratorio de carácter descriptivo, desarrollado por los Centros de Salud Familiar de Sobral - Ceará. De los catorce Centros de Salud investigaron, cuatro fueron con los indicadores apropiados para la atención prenatal. Pero a pesar de que hay deficiencias en la estructura física, no hay impedimentos a fin de proporcionar una calidad de la atención hay una opinión de Que el proceso y los resultados también interferir con la calidad de la atención.

Descriptores: Enfermería; Calidad de atención a la salud; Salud de la família; Atención de Enfermería. 


\section{INTRODUÇÃO}

No Brasil, a atenção à saúde materna e infantil historicamente, tem sido uma prioridade dentre as políticas de saúde, com destaque aos cuidados durante a gestação. A partir dos anos 1970, tal política teve um incremento substancial, devido às elevadas taxas de morbidade e mortalidade materna e infantil, com ampliação da atenção ao pré-natal, face ao reconhecido impacto e transcendência que esta produz no estado sanitário da mãe e do feto.

O cuidado a gestante e ao feto durante o pré-natal, constitui um fenômeno da atenção à saúde ocidental. No modelo de atendimento biomédico ocidental, as mulheres são encorajadas a procurá-lo o mais cedo possível. Essas consultas são geralmente de rotina e, obedecem a uma seqüência sistemática; à consulta inicial seguem-se as mensais, as bimensais e as semanais. A monitorização do peso e da pressão sangüínea, os testes de sangue e urina, as informações específicas sobre dieta, repouso e atividade; e,ainda a preparação para o parto compõe o cuidado pré-natal ${ }^{(1)}$.

O pré-natal é o "período anterior ao nascimento da criança, em Que um conjunto de ações é aplicado à saúde individual e coletiva das mulheres grávidas. Nesse período, as mulheres devem ser acompanhadas a partir da gestação, de forma Que lhes seja possível, Quando necessário, realizar exames clínico-laboratoriais, receber orientação e tomar medicação profilática e/ou vacinas"(2).

Para o Ministério da Saúde do Brasil, o principal objetivo da atenção ao pré-natal é acolher a mulher desde o início de sua gravidez período de mudanças físicas e emocionais - Que cada gestante vivencia de forma distinta. Essas transformações podem gerar medos, dúvidas, angústias, fantasias ou simplesmente a curiosidade de saber o Que acontece no interior de seu corpo ${ }^{(3)}$.

Para promover a segurança da saúde da mãe e do feto é necessário identificar as gestantes de risco e oferecer atendimento diferenciado nos variados graus de exigência, possibilitando a prevenção das complicações Que determinam maior morbidade e mortalidade materna e perinatal. A gravidez é considerada de baixo risco Quando não é necessário aplicar intervenções de maior complexidade e cujas morbidades e mortalidade materna e perinatal são menores do Que as da população geral, ou seja, somente pode ser confirmada ao final do processo gestacional, após o parto e o puerpério. Quando são identificados os fatores associados com pior prognóstico materno e perinatal, a gravidez é definida como de alto risco, passando a exigir avaliações mais freQüentes e com maior complexidade ${ }^{(4)}$.

As ações de saúde desenvolvidas durante a atenção ao pré-natal devem dar cobertura a toda população de gestantes, assegurando o acompanhamento, a continuidade no atendimento e avaliação. Seus objetivos são de prevenir, identificar e/ou corrigir as intercorrências maternas fetais, bem como instruir a gestante no Que diz respeito à gravidez, parto, puerpério e cuidados com o recém-nascido. Destacase, ainda, a importância de oferecer apoio emocional e psicológico ao companheiro e a família, para Que estes também estejam envolvidos com o processo de gestar, parir e nascer.

Buscando melhorar este cenário e, consequentemente, os indicadores de morbidade e mortalidade relacionados à gestação, parto e puerpério, o Ministério da Saúde no ano de 2000 institucionalizou o Programa de Humanização no Pré-natal e Nascimento, com base nos seguintes princípios: "toda gestante tem direito ao acesso a atendimento digno e de Qualidade no decorrer da gestação, parto e puerpério; toda gestante tem direito de saber e ter assegurado o acesso à maternidade em Que será atendida no momento do parto; toda gestante tem direito à assistência ao parto e ao puerpério e que esta seja realizada de forma humanizada e segura, de acordo com os princípios gerais e condições estabelecidas na prática médica; todo recém-nascido tem direito à assistência neonatal de forma humanizada e segura"(5).

O referido programa ${ }^{(5)}$ estabelece como critérios para o cuidado a gestantes e ao bebê o seguinte:

a) Primeira consulta até o $4^{\circ}$ mês de gestação.

b) Garantir a realização dos seguintes procedimentos:

- No mínimo, seis consultas de pré-natal.

- Uma consulta no puerpério, até Quarenta e dois dias após o nascimento.

- Exames laboratoriais;

- Oferta de teste de HIV, com um exame na primeira consulta, naQueles municípios com população maior Que cinqüenta mil habitantes.

- Aplicação de vacina antitetânica até a dose imunizante (segunda) do esquema recomendado, ou dose de reforço em mulheres já imunizadas.

- Atividades educativas.

- Classificação de risco gestacional a ser realizada na primeira consulta e nas consultas subseQüentes.

- Atendimento às gestantes classificadas como de risco, garantindo o vínculo e acesso à unidade de referência para atendimento ambulatorial e/ou hospitalar à gestação de alto risco".

De acordo com a IV Pesquisa de Saúde Materno-Infantil do Ceará - PESMIC IV - 2001, a cobertura da assistência obstétrica, calculada pelo número de gestantes Que fizeram pelo menos uma consulta no pré-natal, foi de $98 \%$ em 200 I , para o estado do Ceará como um todo. Este indicador apresentou uma sensível melhora em relação a 1994, Que apresentava neste período uma cobertura de $84 \%$. Entre as mulheres Que tiveram alguma assistência pré-natal, número médio de consultas foi 6,5 em Fortaleza e 6,3 no interior. A média para o Estado foi de 6,4 consultas por gestante. Em comparação com a PESMIC III, observa-se Que houve um acréscimo no número de consultas do pré-natal no interior do Estado, fato Que pode ser atribuído ao crescente número de equipes do PSF no interior do Estado $^{(6)}$.

Segundo o Ministério da Saúde, as mulheres estão sendo estimuladas a fazer o pré-natal e, respondendo a esse chamado. Elas acreditam Que terão benefícios procurando os serviços de saúde. Depositam sua confiança e entregam seus corpos aos cuidados de pessoas autorizadas legalmente, a cuidarem delas. ${ }^{3}$ Entretanto, o Que se constata é o despreparo ou negligência dos profissionais na adoção de tecnologias de assistência, seja no acompanhamento prénatal ou durante o trabalho de parto.

A organização do serviço tem sido um elemento fundamental para execução da atenção ao pré-natal, pois uma atenção humanizada necessita, além de trabalhadores de saúde Qualificados e sensíveis às necessidades de saúde das famílias, sujeitos e comunidades, como também de ferramentas tecnológicas comuns ao desenvolvimento da consulta, a atenção especializada e a dinâmica do cuidado nos diferentes níveis de atenção e o seguimento desse cuidado de maneira integral e holística. 
Dentro dessa perspectiva surge o interesse pela temática da Qualidade da atenção ao pré-natal em Sobral, em 200 I, Quando foi implantado o Programa de Humanização do Pré-natal e NascimentoPHPN. Apesar do aumento nos índices de consultas, existem muitas situações Que não estão em conformidade com os critérios estabelecidos pelo Ministério da Saúde. Assim, o presente estudo objetiva avaliar a estrutura da atenção ao pré-natal nos territórios da Estratégia Saúde da Família do município de Sobral - Ceará.

Espera-se que esse estudo venha a contribuir para a melhoria da Qualidade na atenção ao pré-natal nos territórios da Estratégia Saúde da Família-ESF do município, possibilitando a indicação de áreas que necessitem de incrementos Quanto ao padrão de Qualidade, sejam elas relacionais, de organização do processo de trabalho ou aQuisição de equipamentos.

\section{METODOLOGIA}

Foi desenvolvida uma pesquisa descritiva-exploratória, em Quatorze (50\%) Centros de Saúde da Família-CSF do município de Sobral - Ceará, durante o período de janeiro a junho de 2007. Em Sobral existem 28 CSF, com 14 na zona rural.

A pesQuisa apresenta-se fundamentada pelo referencial avaliativo proposto por Avedis Donabedian oue traz uma proposta de avaliação em saúde ${ }^{(7)}$. Ele descreve os enfoques que devem ser considerados numa avaliação da Qualidade dos serviços de saúde, afirmando Que independentemente da amplitude da definição de Qualidade, existe um tripé de componentes para avaliá-la, Que é composto por estrutura, processo e resultados.

O termo estrutura faz menção a todos os atributos, materiais e organizacionais, Que são relativamente estáveis no setor Que proporciona a assistência. Já o termo "processo" inclui tudo o que os médicos e outros provedores da atenção fazem por seus pacientes, assim como a habilidade com Que exercem tal assistência. Neste rol também se inclui o Que os pacientes fazem por si mesmos. O termo resultado faz referência ao Que se obtém para o paciente. Mais especificamente, um resultado supõe uma troca no estado de saúde, para o bem ou para o mal, Que pode ser atribuída à assistência. Os resultados também incluem outras características da assistência, por exemplo, conhecimento sobre a enfermidade, trocas na conduta pessoal que conduzem a saúde, e a satisfação do paciente, Que se constitui num elemento particularmente importante, pois em primeiro lugar é um resultado de valor inQuestionável já Que a satisfação é um aspecto de bem-estar Que a atenção intenta promover. Em segundo lugar, a satisfação do paciente contribui para Que ele tenha motivação para uma participação mais efetiva, e em terceiro lugar, a satisfação e o descontentamento constituem os juízos do paciente sobre a Qualidade da atenção recebida e de seus resultados ${ }^{(7)}$.

Como um dos interesses do estudo é o incremento do processo de trabalho do enfermeiro da ESF, estes que ocupavam cargos de gerência nas unidades, foram selecionados como informantes-chave, para responder as perguntas do formulário sobre a estrutura dos CSF. Os enfermeiros foram abordados no próprio CSF, durante o expediente de trabalho. A decisão pela utilização de enfermeiros gerentes se deu por serem os profissionais Que ficam mais à frente da Questão da organização (estrutura, ęuipamentos, insumos, etc). Das Quatorze unidades investigadas apenas em duas não foi possível entrevistar a (o) gerente sendo neste caso, entrevistado (a) um (uma) enfermeiro (a) Que respondia no momento pelo cargo de gerência. Antes da coleta de dados os enfermeiros assinaram o Termo de Consentimento Livre e Esclarecido.

Como técnicas de coleta dos dados foi utilizado um Questionário seguido de observação não-participante. O formulário abordou as seguintes varáveis: I. estrutura física e agendamento; II. eQuipamentos e insumos para semiotécnica; III. material para coleta de exame colpocitológico; apoio laboratorial; Impressos; IV. medicamentos e IV. educação em saúde. Para cada variável foram apontados conceitos de: adeeuado, inadeQuado ou inexistente, de acordo com as normas do Ministério da Saúde. O instrumento de coleta de dados utilizado foi elaborado pela equipe do Trevo de Quatro Folhas de Sobral, juntamente com a Preceptoria de Enfermagem, baseados nos parâmetros do Ministério da Saúde.

Para a realização do estudo foi solicitada autorização junto à Secretaria da Saúde e Ação Social do Município de Sobral-CE, por meio do Núcleo de Estudos e Pesquisas em Saúde-NEPS, tendo sido aprovada. Após, o Protocolo de Pesquisa foi encaminhado ao Comitê de Ética e Pesquisa da Universidade Estadual Vale do Acaraú-UVA, também tendo sido aprovado, com o registro № 392.

\section{RESULTADOS E DISCUSSÃO}

A Qualidade de um serviço de saúde implica na ocorrência de várias circunstâncias específicas e não pode ser determinada por um único fator. Na medida em Que avaliar a Qualidade implica em emitir um juízo de valor, há necessidade de analisarmos seus vários componentes. Na literatura sobre o tema Qualidade em saúde, todos a consideram como um valor complexo Que abrange vários fatores $\mathrm{e}$ ratificam Que uma boa estrutura não determina um padrão de Qualidade, porém boas condições estruturais têm mais possibilidade de resultar em um processo adeQuado de cuidados e em resultado favorável do Que pré-condições precárias ${ }^{(8)}$.

Os dados da Tabela I mostram Que em 93\% dos CSF as gestantes possuem horário específico pré-agendado para sua consulta de prénatal. Tal prática organizativa humaniza a atenção e reduz o tempo de espera da gestante, propiciando conforto e segurança para gestante.

Dos CSF I 4\% não dispõem de pia e $21 \%$ de banheiro. A presença de pia nos consultórios para higienização das mãos e banheiro, permite o controle de infecção e a privacidade da gestante na troca de roupa para realização de exame físico. Segundo o Ministério da Saúde, Dentre os princípios gerais e condições para adequado acompanhamento do pré-natal faz-se necessário uma área física adeQuada para atendimento das gestantes e familiares no Centro de Saúde, em Que a privacidade é um fator essencial para a consulta e para o exame clínico ou ginecológico com condições adęuadas de higiene e ventilaçãa ${ }^{(5)}$.

$\mathrm{O}$ atendimento pré-natal deve ser organizado para atender as reais necessidades de toda a população de gestantes da sua área de atuação por meio da utilização de conhecimentos técnico-científicos e dos meios e recursos adequados disponíveis A estrutura física da instituição deve ser adeQuada e funcional, propiciando condições favoráveis de trabalho aos profissionais e agradáveis às gestantes ${ }^{(9)}$.

Em Sobral, as equipes da ESF possuem mais de um enfermeiro, sendo comum a oferta organizada na atenção pré-natal, e todos agendarem as consultas para um mesmo dia e horário. Sendo que, 
Tabela 1. Avaliação da estrutura física do consultório do pré-natal e do agendamento das consultas. Sobral, Ceará, 2007.

\begin{tabular}{|c|c|c|c|c|}
\hline \multirow{2}{*}{ ESTRUTURA F́́SICA E AGENDAMENTO } & \multicolumn{2}{|c|}{ A } & \multicolumn{2}{|c|}{ I } \\
\hline & $\mathbf{N}$ & $\%$ & $\mathbf{N}$ & $\%$ \\
\hline Gestante possui dia e horário específico para consulta & 13 & 93,0 & 1 & 7,0 \\
\hline Sala de espera com cadeira & 12 & 86,0 & 2 & 14,0 \\
\hline Sala específica e individualizada para consulta & 11 & 79,0 & 3 & 21,0 \\
\hline Cada consultório dispõe de pia para lavar as mãos & 12 & 86,0 & 2 & 14,0 \\
\hline Cada consultório dispõe de um banheiro & 11 & 79,0 & 3 & 21,0 \\
\hline $\begin{array}{l}\text { Há sistema para regular a temperatura do consultório } \\
\text { Há sala disponível para atividades docentes e/ou edu cação em }\end{array}$ & 14 & 100,0 & - & - \\
\hline saúde* & 10 & 71,0 & 1 & 7,0 \\
\hline
\end{tabular}

nem sempre o CSF dispõe de consultórios em número suficiente para todos os profissionais, assim sendo, dois enfermeiros atendem gestantes em um mesmo consultório. É importante ressaltar que a Consulta de Enfermagem no pré-natal ou não, sendo realizada em sala comum por dois enfermeiros, gera algumas dificuldades para o processo de trabalho, tais como: o ruído de comunicação, pelas falas das enfermeiras simultaneamente ou das gestantes; interrupções, ora pelo auxiliar de enfermagem, Que traz os prontuários, ora por gestante Querendo ser atendida "só para tirar uma dúvida", além da entrada de outros profissionais com problemas administrativos, para Que uma das enfermeiras fosse resolvê-los; além da ausência de privacidade das gestantes e descontinuidade do ritmo da consulta.

Uma boa ambiência segundo a Política Nacional de Humanização, compreende o "ambiente físico, social, profissional e de relações interpessoais Que deve estar relacionado a um projeto de saúde voltado para a atenção acolhedora, resolutiva e humana”. A ambiência, segue os seguintes eixos norteadores: $\mathrm{O}$ espaço Que possibilita a reflexão da produção do sujeito e do processo de trabalho. O espaço Que visa à confortabilidade focada na privacidade e individualidade dos sujeitos envolvidos, exaltando elementos do ambiente Que interagem com o homem - a dizer cor, cheiro, som, iluminação, morfologia -, e garantindo conforto a trabalhadores, cliente e sua rede social. O espaço como ferramenta facilitadora do processo de trabalho funcional, favorecendo a otimização de recursos e o atendimento humanizado, acolhedor e resolutivo(10).

Os resultados apontam Que 14\% dos CSF não possuem espaço para atividades de educação em saúde. As atividades de comunicação/ informação em saúde devem ser priorizadas no transcurso da atenção ao pré-natal, uma vez Que o intercâmbio de informações e experiências pode ser a melhor forma de promover a compreensão do processo de gestar e repassar orientações/informações às gestantes, incluindo, também, seu companheiro e familiares ${ }^{(5)}$.

Conforme o Ministério da Saúde é necessário que o setor "Saúde esteja aberto para as mudanças sociais e cumpra de maneira mais ampla o seu papel de educador e promotor da saúde. As gestantes constituem o foco principal do processo de aprendizagem, porém não se pode deixar de atuar, também, entre companheiros e familiares. A posição do homem na sociedade está mudando tanto Quanto os papéis tradicionalmente atribuídos às mulheres. Portanto, os serviços devem promover o envolvimento dos homens, adultos e adolescentes, discutindo a sua participação responsável nas Questões da saúde sexual e reprodutiva”(11).

Segundo Rios e Vieiraa ${ }^{(12)}$, a "realização de ações educativas no decorrer de todas as etapas do ciclo grávido-puerperal é muito importante, mas é o pré-natal Que a mulher deverá ser melhor orientada para Que possa viver o parto de forma positiva, ter menos riscos de complicações no puerpério e mais sucesso na amamentação. Considerando o pré-natal e nascimento como momentos únicos para cada mulher e uma experiência especial no universo feminino, os profissionais de saúde devem assumir a postura de educadores Que compartilham saberes, buscando devolver à mulher sua autoconfiança para viver a gestação, o parto e o puerpério".

A Tabela 2 mostra Que, a maioria dos CSF estão providos de equipamentos e insumos mínimos, necessários para a realização de um exame físico adequado, não estando fora dos padrões exigidos pelos Ministérios da Saúde, Que de acordo com o Programa de Humanização do Pré-natal e Nascimento preconiza Que deve ser garantido a existência de equipamentos e instrumental mínimos para a realização das consultas de pré-natal(5).

Com a densidade tecnológica disponível no mercado sanitário, vem ocorrendo uma intensa mecanização da atenção ao pré-natal, onde ocorre uma progressiva substituição da clássica consulta (com a utilização da semiologia e semiotécnica) baseada em tecnologias leves e leve-duras, por um simples diagnóstico por imagem, com o uso exacerbado de tecnologias duras, levando a inversão da atenção carregada por trabalho vivo para um modelo centrado no trabalho morto, em Que não são priorizadas as relações, a afetividade e a troca de subjetividades, fato Que contribui significativamente para a não humanização desta atenção.

A realização do exame físico geral, com a medição da altura uterina dentre outros, permite o toQue e uma maior aproximação profissionalgestante, por conseguinte, corroborando com o estabelecimento do plano de cuidados e uma gestação saudável.

Dentre os exames básicos preconizados pelo Ministério da Saúde por meio do PHPN está o exame de colpocitologia oncótica Que, para sua realização necessita de alguns equipamentos e insumos específicos dentre os Quais estão o biombo e óculos de proteção individual(5).

O uso do biombo está relacionado à privacidade da gestante. Os óculos compoem a lista de eQuipamentos de proteção individual-EPI profissional, essencial na realização dos exames ginecológicos, tanto Quanto a máscara e as luvas. Em Sobral, das Quatorze unidades investigadas, em apenas duas existiam de óculos de proteção. O uso de EPI durante procedimentos invasivos ou Que permitam o contato com soluções corporais, permite a garantia da segurança para o profissional e a cliente, assim como a Qualidade da atenção. Duas 
Tabela 2. Equipamentos e insumos da semiotécnica do pré-natal disponíveis. Sobral, Ceará, 2007.

\begin{tabular}{|c|c|c|c|c|}
\hline \multirow{2}{*}{ EQUIPAMENTOS E INSUMOS } & \multicolumn{2}{|c|}{ A } & \multicolumn{2}{|c|}{ I } \\
\hline & $\mathbf{N}$ & $\%$ & $\mathbf{N}$ & $\%$ \\
\hline Mesa e cadeiras para entrevista & 13 & 93,0 & 1 & 7,0 \\
\hline Mesa de exame ginecológico & 12 & 86,0 & 2 & 14,0 \\
\hline Escada de dois degraus & 13 & 93,0 & 1 & 7,0 \\
\hline Foco de luz & 13 & 93,0 & 1 & 7,0 \\
\hline Balança antropométrica adulto & 14 & 100,0 & - & - \\
\hline Esfignomanômetro & 14 & 100,0 & - & - \\
\hline Estetoscópio clínico & 14 & 100,0 & - & - \\
\hline Estetoscópio de Pinard & 14 & 100,0 & - & - \\
\hline Sonar Doppler & 14 & 100,0 & - & - \\
\hline Fita métrica flexível e inelástica & 14 & 100,0 & - & - \\
\hline Gestograma ou disco obstétrico & 14 & 100,0 & - & - \\
\hline Nomograma & 14 & 100,0 & - & - \\
\hline Álcool 70\% com algodão & 14 & 100,0 & - & - \\
\hline Papel toalha & 13 & 93,0 & 1 & 7,0 \\
\hline Gel para sonar & 14 & 100,0 & - & - \\
\hline Água para lavagem das mãos & 14 & 100,0 & - & - \\
\hline Sabão para lavagem das mãos & 14 & 100,0 & - & - \\
\hline
\end{tabular}

enfermeiras relatam não possuir o biombo no CSF, deixando suas gestante sem nenhuma privacidade durante as consultas e exames ginecológicos.

Para realização de um adeQuado acompanhamento pré-natal e assistência às gestantes e à puérpera, o município deverá, por meio das unidades integrantes da rede de atenção à saúde, garantir a realização dos exames básicos o mais próximo possível do território em Que moram estas. O plano operacional da PHPN definiu elementos chaves da assistência à gestação e ao parto, em torno dos Quais deveria concentrar esforços a fim de alcançar o objetivo principal de reduzir as altas taxas de morbi-mortalidade materna e perinatal $\left.\right|^{(5)}$. sendo a garantia dos exames básicos uma destas ações.

A garantia de exames básicos Que diagnosticam ou previnem agravos/doenças como anemias, sífilis congênita, aids, infecção urinária dentre outros são essenciais para uma satisfatória atenção ao pré-natal e Qualidade de vida materna e infantil, permitindo a redução dos riscos de aquisição de morbidades e mortalidade perinatal e neonatal.

O PHPN instituiu ainda uma estratégia para induzir e auxiliar a maioria dos municípios a implementar essas ações, introduzindo novos recursos para o custeio dessa assistência e transferindo-os mediante o cumprimento de critérios mínimos, necessários para melhorar a Qualidade da assistência. Dentre os critérios recomendados foram: exames laboratoriais: (a) ABO-Rh, na primeira consulta; (b) VDRL, um exame na primeira consulta e outro próximo à trigésima semana da gestação; (c) Urina rotina, um exame na primeira consulta e outro próximo à trigésima semana da gestação; (d) Glicemia de jejum, um exame na primeira consulta e outro próximo à trigésima semana da gestação; (e) Hemoglobina/Hematócrito, na primeira consulta; oferta de testagem anti-HIV, com um exame na primeira consulta, naQueles municípios com população acima de cin̨üenta mil habitantes ${ }^{(5)}$.

De acordo com os resultados identificados no Que se refere à realização de exames, os CSF de Sobral vêm funcionando de forma satisfatória. Visto Que dentre as mínimas deficiências estão à dificuldade da solicitação de urocultura com antibiograma e proteinúria apontadas como inadequadas em dois CSF, Que precisam ser autorizadas para serem realizados em laboratórios particulares e, deficiência Quanto ao teste anti-HIV, Que foi o mais apontado entre os exames como inadeQuado, pelo fato de seu resultado chegar muitas vezes depois de a gestante ter parido.

Segundo a Federação Brasileira de Ginecologia e ObstetríciaFEBRASGO para Que o acompanhamento da gestante seja efetivo, a estrutura da unidade precisa, ainda, permitir o acesso aos exames laboratoriais especializados, Quando pertinentes ${ }^{(9)}$.

No Que se refere á disponibilidade de medicamenos, os resultados apontam que em três CSF faltam medicamentos para o tratamento de Doenças Sexualmente Transmissíveis-DST. Alguns enfermeiros entrevistados argumentaram Que só existe um tipo de medicação e Que muitas vezes a gestante necessita ser tratada com uma outra medicação Que não está disponível na unidade, e por não ter condiçõos para comprar, ela acaba tendo infecção vaginal durante todo o período da gestacional, Que dependendo da doença, pode elevar os riscos de mortalidade neonatal.

Quanto à magnitude das DST, a Organização Mundial de SaúdeOMS estimou em 1999, a existência de 340 milhões de novos casos/ano de DST curáveis em todo o mundo, entre sujeitos de 15 e 49 anos, sendo de 10 a 12 milhões destes casos no Brasil. Se imaginarmos a multiplicidade de infecções/sujeito e a presença de infecções de outras DST virais crônicas, poderemos perceber a magnitude do problema. A mulher, mais do Que nunca, é peça fundamental no controle das DST. E, ao fazer o pré-natal, necessita de orientações adeQuadas para melhor tratamento clínico e preventivo das DST com melhor assistência também ao concepto. É necessário interromper a cadeia de transmissão oportunamente e prevenir novas ocorrências ${ }^{(15)}$.

Quanto às ações de educação em saúde desenvolvidas durante o pré-natal, dos enfermeiros investigados, dois classificaram seus grupos de gestantes como inadeQuados. Eles explicam Que apesar de não possuírem um grupo organizado, as gestantes recebem algumas 
informações por meio de palestras enQuanto esperam atendimento.

Quanto as práticas educativas com gestantes e familiares o Ministério da Saúde destacam as discussões realizadas em grupos, com trocas de experiências entre os componentes do grupo, além de dramatizações. O profissional de saúde, atuando como facilitador, deve evitar o estilo palestra, pouco produtiva, Que ofusca Questões subjacentes, na maioria das vezes, mais importantes ${ }^{(11)}$. As palestras muitas vezes não problematizam os temas relacionados ao período perinatal e percebe-se em vivências, Que a linguagem utilizada é técnica e não a coloquial própria da comunidade, o Que pode dificultar o entendimento ou produzir ruídos de comunicação.

Como a gestação é um período Que envolve grandes mudanças bio-psico-sociais, gestantes e seus pares necessitam compartilhar reflexões sobre as mudanças Que vivenciam, trocar informações objetivas sobre a experiência de parto e pós-parto, bem como se preparar do ponto de vista corporal e emocional para as experiências Que viverão.

O período pré-natal para Rios e Vieira ${ }^{(12)}$ é uma época de preparação física e psicológica para o parto e para a maternidade e, como tal, é um momento de intenso aprendizado e uma oportunidade para os profissionais da equipe de saúde desenvolverem a educação como dimensão do processo de cuidar.

Viçosa ${ }^{(16)}$ contribui referindo Que nos grupos de gestantes se cria um espaço onde os participantes podem dizer seus problemas e refletir sobre eles. Nesta perspectiva há o incentivo para a troca de experiências comuns entre os integrantes e os coordenadores do grupo, o Que gera mobilização entre os participantes.

No que tange aos indicadores de resultado Donabedian afirma Que a avaliação de resultados inclui: alterações de ordem clínica, psicológica-bioquímica, física, psicológica (mental), social e psicológica, resultados integrados e resultados avaliativos ${ }^{(7)}$. Hoje, os principais caminhos para desenvolver a abordagem de resultado passam pelo desenvolvimento de indicadores ou índices do nível de saúde e pela medida da satisfação do cliente. Porém, na prática, resultados são difíceis de medir. A ocorrência do impacto final da atenção pode levar um tempo muito longo, pois falta um padrão estabelecido com que se possa medir o resultado observado.

Diante do exposto, os indicadores de resultados podem retratar a eficiência da assistência pré-natal e analisar os indicadores de saúde expressos nos Sistema de Informação da Atenção Básica-SIAB, Sistema de Informação sobre Mortalidade-SIM, SISPRENATAL, Sistema de Informação de Nascidos Vivos-SINASC e Sistema de Informação sobre Agravos Notificáveis-SINAN.

Optamos então pelos seguintes indicadores de resultados integrados: coeficiente de mortalidade materna; coeficiente de mortalidade infantil. Os coeficientes de mortalidade materna e infantil são influenciados pelas condições de assistência ao pré-natal e ao parto, bem como pelos aspectos biológicos da reprodução humana e pela presença de doenças provocadas ou agravadas pelo ciclo gravídico puerperal. Cerca de $98 \%$ das mortes de mulheres por causas maternas são evitáveis, mediante a adoção de medidas relativamente simples, visando a melhorar a Qualidade da assistência pré-natal e garantir o acesso aos serviços de saúde ${ }^{(16)}$.

No Que se refere aos resultados avaliativos foram considerados: percentual de gestantes com seis ou mais consultas de pré-natal; percentual de gestantes Que realizaram seis consultas de pré-natal e todos os exames básicos. Também foram considerados resultados clínicos como: percentual de recém-nascidos com diagnóstico de sífilis congênita; percentual de recém-nascidos com tétano neonatal.

Conforme já mencionamos, os dados coletados para esta fase foram provenientes de indicadores epidemiológicos Que retratam o impacto da Qualidade do pré-natal do município de Sobral no ano de 2006. Assim, buscamos junto à Vigilância Epidemiológica do município os indicadores acima mencionados.

Os dados foram organizados em um Quadro apontando a "estrutura" de cada Centro de Saúde da Família. Quanto ao item "resultado" este foi apresentado através de uma tabela demonstrado o desempenho da Qualidade da assistência pré-natal na sede do município de Sobral.

A análise deu-se comparando os resultados da investigação da "estrutura" com os indicadores epidemiológicos que avaliam o item "resultado".

De acordo com a disponibilidade dos dados, foi possível demonstrar seis indicadores de resultados utilizados no estudo: coeficiente de mortalidade materna; coeficiente de mortalidade infantil, percentual de gestantes com seis ou mais consultas de pré-

\begin{tabular}{|c|c|c|c|c|c|c|c|c|c|c|c|c|c|c|}
\hline \multirow{2}{*}{ Indicadores } & \multicolumn{14}{|c|}{ Unidades } \\
\hline & A & B & $\mathrm{C}$ & D & $E$ & $\mathrm{~F}$ & G & $\mathrm{H}$ & $I$ & $\mathrm{I}$ & $\mathbf{L}$ & $\mathrm{M}$ & $\mathrm{N}$ & 0 \\
\hline $\mathrm{CMM}$ & 1,1 & - & - & - & - & - & 0,6 & - & - & - & - & - & - & - \\
\hline CMI & - & - & - & - & - & - & - & - & - & - & - & - & - & - \\
\hline CMP & - & - & - & - & - & - & - & - & - & - & - & - & - & - \\
\hline PG6CPN & 87,8 & 78,1 & 89,0 & 94,7 & 88,5 & 92,7 & 82,8 & 84,8 & 85,1 & 84,7 & 94,7 & 73,3 & 87,8 & 86,0 \\
\hline PG6PNEB & 82,9 & 74,5 & 87,3 & 94,7 & 82,8 & 87,2 & 82,8 & 81,8 & 81,4 & 82,6 & 82,7 & 78,3 & 85,0 & 86,0 \\
\hline PRNSC & - & - & - & - & - & - & 0,6 & - & - & - & - & - & 0,34 & - \\
\hline PRNTN & - & - & - & - & - & - & - & - & - & - & - & - & - & - \\
\hline
\end{tabular}

Legenda: CMM: Coeficiente de Mortalidade Materna (/I00.000 nascidos vivos)

CMI: Coeficiente de Mortalidade Infantil (/I.000 nascidos vivos)

CMP: Coeficiente de Mortalidade Perinatal (/I.000 nascidos vivos)

PG6CPN: Percentual de gestantes Que realizaram seis ou mais consultas de pré-natal

PG6PNEB: Percentual de gestantes Que realizaram seis ou mais consultas e todos os exames básicos

PRNSC: Percentual de recém-nascidos com diagnósticos de sífilis congênita

PRNTN: Percentual de recém-nascidos com diagnósticos de tétano neonatal

Quadro 1. Indicadores epidemiológicos da Qualidade da assistência pré-natal na sede de Sobral, Ceará, 2007. 
natal; percentual de gestantes Que realizaram seis consultas de prénatal e todos os exames básicos; percentual de recém-nascidos com diagnóstico de sífilis congênita e percentual de recém-nascidos com tétano neonatal, considerando-se Que todos são indicadores de Qualidade de assistência à mulher durante a gestação, parto e puerpério.

Indicadores de saúde são parâmetros utilizados intencionalmente como o objetivo de avaliar, sob o ponto de vista sanitário, a higidez de agregados humanos, bem como fornecer subsídios aos planejamentos de saúde, permitindo o acompanhamento das flutuações e tendências históricas do padrão sanitário de diferentes coletividades consideradas à mesma época ou da mesma coletividade em diversos períodos de tempo ${ }^{(19)}$.

A utilização de indicadores de saúde permite o estabelecimento de padrões, bem como o acompanhamento de sua evolução ao longo dos anos. Embora o uso de um único indicador isoladamente não possibilite o conhecimento da complexidade da realidade social, a associação de vários e, ainda, a comparação entre diferentes indicadores de distintas localidades facilita sua compreensão ${ }^{(18)}$.

No Quadro VII vimos os indicadores de resultados da assistência pré-natal prestada pelas equipes na Estratégia Saúde da Família da sede de Sobral em 2006.

O Coeficiente de Mortalidade Materna (CMM) é um indicador extremamente fiel para aferirmos a situação das mulheres em um dado momento em um determinado país. Daí a importância de destacálo entre os indicadores utilizados na avaliação da assistência à mulher, cuja magnitude do problema tem sido tema de diversas pesquisas nos últimos 15 anos $^{(19)}$.

Se considerarmos o CMM neste estudo, Sobral não está fora das drásticas estatísticas do Brasil, onde segundo dados do Ministério da Saúde, morrem anualmente duas mil mulheres por complicações na gravidez, parto ou aborto e vem se mantendo em patamar elevado nos últimos 2 anos $^{(15)}$.

Os óbitos infantis, mesmo em número pequeno, estão presentes em nove dos Quatorzes Centros de Saúde. Os números mostram também, Que os óbitos perinatais estão presentes na metade das unidades de saúde.

O Coeficiente de Mortalidade Infantil é reconhecido como um dos mais sensíveis indicadores de saúde, pois a morte de crianças menores de um ano é diretamente influenciada por condições de pré-natal, gravidez, história materna, conduta e doenças maternas, ruptura precoce de membrana, gemelaridade, idade materna, condições e tipo de parto, etc. Conhecer o perfil da mortalidade infantil é fundamental para a formulação de estratégias Que permitam o seu controle, este deve ser feito desde uma assistência adequada à mulher durante $\mathrm{a}$ gravide $\mathrm{z}^{(18)}$.

No presente estudo, vimos Que o CMI está sob controle, visto Que esse índice encontra-se num patamar baixo e estável entre os Centros de Saúde aqui analisados.

No Brasil, a mortalidade perinatal é três vezes maior (30/1. 000 para o ano de 1986) do Que a dos países desenvolvidos, onde os óbitos perinatais estão basicamente ligados a problemas genéticos, anomalias congênitas, erros inatos do metabolismo e outras causas Que fogem do sistema de saúde ${ }^{(19)}$.

O Coeficiente de Mortalidade Perinatal dos Centros de Saúde da sede de Sobral é um dos fatores de influência na mortalidade infantil e nos mostram Que o número de natimortos está ainda muito elevado, influenciando no alto índice de mortalidade perinatal.

Os indicadores de consulta de pré-natal e exames básicos estão numa faixa de 70 a $90 \%$ nos Centros de Saúde analisados. Os Percentuais de Consultas e Exames Básicos demonstram Que, nos Centros de Saúde da sede de Sobral, esses números ainda estão pequenos uma média de 78 a 94\%, visto Que todas essas áreas têm $100 \%$ de cobertura e no Quadro de avaliação de estrutura os exames laboratoriais básicos são considerados adeQuados por todos os catorze Centros de Saúde analisados.

Com relação ao percentual de recém-nascidos com diagnóstico de sífilis congênita, Sobral aponta este indicador negativo em dois dos Centros de Saúde aqui estudado.

Em 1993, o Ministério da Saúde, ciente dos riscos e da magnitude da Sífilis Congênita no Brasil, propôs a sua erradicação no país até o ano de 2000, visto ser esta uma entidade clínica totalmente passível de prevenção por meio da identificação e tratamento das gestantes infectadas ainda no pré-natal.

Entretanto, as metas governamentais até agora não foram atingidas e, de acordo com informes oficiais do próprio governo brasileiro, as taxas de Sífilis Congênita ainda permanecem extremamente elevadas, como mostram os números neste estudo. Desse modo, merece reflexão a prevalência de SC no presente estudo, uma vez que a grande maioria das gestantes tem acesso à assistência pré-natal e ainda assim observou-se uma taxa de SC extremamente elevada.

Tétano neonatal é a segunda maior freqüência de mortalidade entre as seis infecções preveníveis por vacinas em países em desenvolvimento. Em 1998 a Assembléia Mundial da Saúde/0MS) aprovou resolução para eliminar o tétano neonatal (TNN) do mundo até o ano de 1995. O Conselho Diretor da Organização PanAmericana da Saúde (CD/OPAS) estabeleceu a estratégia neste mesmo ano. Para alcançar essa meta a OPAS estabeleceu a estratégia de vacinação de 1005 de mulheres em idade fértil (MIF) de 15 a 49 anos Que vivem em área de risco para TNN.

Em Sobral, de acordo com nosso estudo, a meta de erradicação do TNN foi alcançada, visto Que nenhum caso da doença é confirmado há cinco anos.

\section{CONSIDERAÇÕES FINAIS}

Em resumo, a avaliação da Qualidade demonstrou ser um poderoso instrumento de identificação de pontos fortes e de pontos fracos na estrutura e resultado da assistência pré-natal. Se por um lado demonstrou-se que alguns Centros de Saúde atingiram indicadores satisfatórios em alguns itens, por ouro, outros Centros de Saúde mostraram suas deficiências.

Entretanto dos Quatorze Centros de Saúde investigados, Quatro se mostraram com indicadores adequados para o pré-natal. Vale salientar Que entre esses Quatro, um foi o Que mais apontou inadeQuação na sua estrutura, o Que deixa claro Que a estrutura física inadęuada não impede Que se ofereça um atendimento de Qualidade, Que pode estar na forma como acolhemos e damos resolubilidade aos problemas das nossas gestantes.

Enfim, foram evidenciadas algumas deficiências na estrutura Que precisam de investimentos para serem resolvidas. E uma das limitações do presente trabalho se refere aos indicadores de resultado: é muito difícil se associar maior mortalidade à baixa Qualidade. Como exemplo disso, podemos verificar que o Centro de Saúde que apresentou 
todos os coeficientes de mortalidade e ainda o percentual de sífilis congênita, classificou todos os itens de avaliação de estrutura como adequado. Porém possui uma área sem cobertura de ACS que é composta por uma classe elitizada, a Qual nós sabemos, é a mais resistente ao pré-natal no SUS. A Questão social não deve ser esQuecida Quando analisamos indicadores, pois sabemos Que alguns Centros de Saúde de Sobral cobrem áreas Que são de risco social extremo e que por isso muitas vezes a existência de alguns indicadores ruins chega a ser Quase inevitável.

Apesar das limitações inerentes a uma avaliação de Qualidade, foi possível evidenciar Que Sobral vem melhorando muito a atenção ao pré-natal. Hoje com a Preceptoria de Enfermagem e a educação permanente para os enfermeiros da Estratégia Saúde da Família com uma equipe de multiprofissionais Que direcionam seus olhares para as dificuldades que possam surgir no âmbito da saúde materno infantil, fica mais fácil evitar o surgimento de indicadores negativos, visto Que o suporte desses profissionais é de grande valia para a enfermagem nas equipes de saúde da família.

Compreendendo as inúmeras dificuldades e desafios que se colocam no atendimento à saúde, espera-se Que essas informações sirvam de estímulo às equipes de saúde para o desenvolvimento de iniciativas de melhoria de Qualidade das condições que resultem em indicadores satisfatórios, e Que os mesmos assim possam ser mantidos.

\section{REFERÊNCIAS}

I. Lowdermilk DL, Perry SE, Bobaki IM. O cuidado em enfermagem materna. $5^{\mathrm{a}}$ ed. Porto Alegre: Artmed; 2002.

2. Ministério da Saúde (BR). Secretaria-Executiva. Secretaria de Vigilância em Saúde. Glossário temático: DST e AIDS. Brasília: Ministério da Saúde; 2006.

3. Ministério da Saúde (BR). Secretaria de Políticas de Saúde. Área Técnica da Saúde da Mulher. Assistência pré-natal: manual técnico. $3^{\text {a }}$ ed. Brasília: Ministério da Saúde; 2000.

4. Gaio DSM. Assistência pré-natal e puerpério. In: Duncan BB, Schmidt MI, Giugliani ERJ, colaboradores. Medicina ambulatorial - condutas de atenção primária baseadas em evidências. $3^{\text {a }}$ ed. Porto Alegre: Artmed; 2004. p. 357-67.

5. Ministério da Saúde (BR). Programa de humanização no prénatal e nascimento. Brasília: Ministério da Saúde; 2000.

6. Secretaria de Estado da Saúde do Ceará. Saúde reprodutiva e sexual: um manual para atenção primária e secundária (nível ambulatorial). Fortaleza: SESA; 2002.

7. Donabedian A. An introduction to Quality assurance in health care. New York: Oxford; 2003.

8. Alves MTSB, Silva AAM, organizadoras. Avaliação de Qualidade de maternidades: assistência à mulher a ao seu recém-nascido no sistema único de saúde. São Luis: UFMA; 2000.

9. Federação Brasileira de Ginecologia e Obstetrícia. Assistência Pré-natal: manual de orientação. São Paulo: FEBRASGO; 2005.

10. Ministério da Saúde (BR). Política Nacional de Humanização HumanizaSUS: documento base para gestores e trabalhadores do SUS. Brasília: Ministério da Saúde; 2004.

11. Ministério da Saúde (BR). Pré-natal e puerpério - Atenção Qualificada e humanizada. $5^{\mathrm{a}}$ ed. Brasília: Ministério da Saúde; 2005.

12. Rios CTF, Vieira NFC. Ações educativas no pré-natal: reflexão sobre a consulta de enfermagem como um espaço para educação em saúde. Ciência \& Saúde Coletiva 2007; 12(2): 477-86.

13. Ministério da Saúde(BR). Secretaria de Vigilância em Saúde. Programa Nacional de DST e Aids. Manual de Controle das DST. $4^{a}$ ed. Brasília: Ministério da Saúde; 2005.

14. Viçosa GR. Grupos com gestantes. Porto Alegre: Artes Médicas; 1997.

15. Batista V. Realização do pré-natal e boa assistência ao parto reduzem mortalidade de mães e bebês. [citado em 2 l jan 2008]. Disponível em: http://portalweb02.saúde.gov.br

16. Bem-Estar Familiar Brasil. PesQuisa nacional sobre demografia e saúde, 1996. Rio de Janeiro; 2008. [citado em 2 I jan 2008]. Disponível em: http://www.bemfam.org.br/info_publicacoes.php.

17. Roquayrol MZ. Epidemiologia e saúde. Rio de Janeiro: MEDSI; 1993.

18. Secretaria da Saúde do Ceará. Escola de Saúde Pública do Ceará. Curso básico de sistemas de informação. Fortaleza: Secretaria da Saúde do Ceará; 2001 .

19. Ducan BD, Schimidt MI, Gigliani ERJ. Medicina ambulatorial: condutas clínicas em atenção primária. $2^{\mathrm{a}}$ ed. Porto Alegre: Artes Médicas; 1996. 